PROCEEDINGS OF THE

AMERICAN MATHEMATICAL SOCIETY

Volume 129, Number 9, Pages 2563-2570

S 0002-9939(01)05840-3

Article electronically published on February 9, 2001

\title{
ON THE REGULARITY OF $p$-BOREL IDEALS
}

\author{
JÜRGEN HERZOG AND DORIN POPESCU
}

(Communicated by Wolmer V. Vasconcelos)

\begin{abstract}
In this paper we prove Pardue's conjecture on the regularity of principal $p$-Borel ideals. As a consequence we obtain an upper bound for the regularity of general $p$-Borel ideals.
\end{abstract}

\section{INTRODUCTION}

Let $K$ be field and $I \subset S$ a graded ideal in the polynomial ring $S=K\left[x_{1}, \ldots, x_{n}\right]$. Recall that the generic initial ideal $\operatorname{Gin}(I)$ of $I$ with respect to the reverse lexicographical order is Borel-fixed. This means that Gin $(I)$ is fixed under the action of the Borel group of the upper triangular invertible matrices acting linearly on the polynomial ring. By a theorem of Bayer and Stillman 2], the regularity of $I$ and $\operatorname{Gin}(I)$ coincide. This is one of the reasons why one is interested in computing the regularity of $\operatorname{Gin}(I)$. In characteristic zero a Borel-fixed ideal is strongly stable, and so its regularity is simply the highest degree of a minimal generator. In positive characteristic however, Borel-fixed ideals are $p$-Borel (see 1.1 for the definition), and these are monomial ideals with a quite difficult combinatorial structure.

Monomials $u_{1}, \ldots, u_{m} \in I$ of a $p$-Borel are called Borel generators of $I$ if $I$ is the smallest $p$-Borel ideal containing $u_{1}, \ldots, u_{m}$. In this case we write $I=$ $\left\langle u_{1}, \ldots, u_{m}\right\rangle$. The ideal $I$ is called principal $p$-Borel if $I$ has only one Borel generator. Pardue conjectured a formula for the regularity of a principal $p$-Borel ideal which only depends on the exponents of the Borel generator (see 1.4). In a paper by Aramova and Herzog [1] it was shown that Pardue's formula indeed gives a lower bound for the regularity. Some of the results in that paper were later extended by Ene, Pfister and Popescu 5 to more general ideals. In the present paper we will show that Pardue's formula also yields an upper bound. Our method in proving this uses a criterion of Eisenbud, Reeves and Totaro [4 for determining the regularity of $p$-Borel ideals.

The authors are grateful to the Alexander von Humboldt Foundation for supporting their collaboration.

\section{1. $p$-BOREL IDEALS}

Throughout this paper we fix a field $K$ and let $S=K\left[x_{1}, \ldots, x_{n}\right]$ be the polynomial in $n$ indeterminates over $K$.

Received by the editors October 12, 1999 and, in revised form, January 24, 2000.

1991 Mathematics Subject Classification. Primary 13P10; Secondary 13D02, 13C13.

Key words and phrases. Regularity, $p$-Borel ideals. 
Let $p$ be a prime number, and $k$ and $l$ be non-negative integers with $p$-adic expansion $k=\sum_{i} k_{i} p^{i}$ and $l=\sum_{i} l_{i} p^{i}$. We set $k \leq_{p} l$ if $k_{i} \leq l_{i}$ for all $i$.

Definition 1.1. A monomial ideal $I \subset S$ is $p$-Borel if the following condition holds: for each monomial $u \in I, u=\prod_{i} x_{i}^{\mu_{i}}$, one has $\left(x_{i} / x_{j}\right)^{\nu} u \in I$ for all $i, j$ with $1 \leq i<j \leq n$ and all $\nu \leq_{p} \mu_{j}$.

The significance of $p$-Borel principal ideals is given by

Proposition 1.2 (Pardue). Suppose char $K=p$, and let $I \subset S$ be a monomial ideal. Then $I$ is Borel-fixed if and only if $I$ is p-Borel.

We denote by $G(I)$ the unique minimal set of monomial generators of a monomial ideal $I$. It is easy to see (cf. [1]) that $I$ is $p$-Borel if the conditions of 1.1 are satisfied for all $u \in G(I)$.

A principal $p$-Borel ideal can be explicitly described. We use the following standard notation: If $J$ is a monomial ideal we let $J^{\left[p^{i}\right]}$ be the ideal generated by all monomials $u^{p^{i}}$ with $u \in G(J)$. The ideal $J^{\left[p^{i}\right]}$ is called the $p^{i}$ th Frobenius power of $J$. Note that we define the $p^{i}$ th Frobenius power of monomial ideals in any characteristic.

Proposition 1.3 (Pardue). Let $u=\prod_{i} x_{i}^{\mu_{i}}$, and let $\mu_{i}=\sum_{i} \mu_{i j} p^{j}$ be the $p$-adic expansion of $\mu_{i}$ for $i=1, \ldots, n$. Then

$$
\langle u\rangle=\prod_{i=1}^{n} \prod_{j}\left(\left(x_{1}, \ldots, x_{i}\right)^{\mu_{i j}}\right)^{\left[p^{j}\right]} .
$$

In particular, $\langle u\rangle=\prod_{i=1}^{n}\left\langle x_{i}^{\mu_{i}}\right\rangle$.

It follows from 1.3 that $\left\langle x^{\mu}\right\rangle=\left(x_{1}^{\mu_{1}}\right)\left\langle x^{\mu} / x_{1}^{\mu_{1}}\right\rangle$, so that $\operatorname{reg}\left\langle x^{\mu}\right\rangle=\mu_{1}+\operatorname{reg}\left\langle x^{\mu} / x_{1}^{\mu_{1}}\right\rangle$. Therefore, if we are interested in the regularity of the $p$-Borel principal ideal $\left\langle x^{\mu}\right\rangle$ we may assume that $x_{1}$ does not divide $x^{\mu}$.

Denote by $\lfloor *\rfloor$ the greatest integer function, and for $1 \leq k \leq n$ and $j \geq 0$ define

$$
d_{k j}(\mu)=\sum_{i=1}^{k}\left\lfloor\frac{\mu_{i}}{p^{j}}\right\rfloor .
$$

For each $k$ such that $\mu_{k} \neq 0$, let $s_{k}=\left\lfloor\log _{p} \mu_{k}\right\rfloor$, and set

$$
D_{k}=d_{k s_{k}}(\mu) p^{s_{k}}+(k-1)\left(p^{s_{k}}-1\right) .
$$

Conjecture 1.4 (Pardue). If $x_{1}$ does not divide $x^{\mu}$, then

$$
\operatorname{reg}\left\langle x^{\mu}\right\rangle=\max _{k: \mu_{k} \neq 0}\left\{D_{k}\right\} .
$$

In the following we will express the right-hand side of Conjecture 1.4 in different ways. The following easy fact can be found in [1].

Proposition 1.5. Let $\mathcal{S}=\left\{s_{k}: \mu_{k} \neq 0\right\}$, let $q_{j}=\max \left\{k: s_{k}=j\right\}$ for each $j \in \mathcal{S}$, and set $E_{j}=D_{q_{j}}$. Then:

(i) $E_{j}=\sum_{i=j}^{s}\left(\sum_{k=2}^{q_{j}} \mu_{k i}\right) p^{i}+\left(q_{j}-1\right)\left(p^{j}-1\right)$ for all $j \in \mathcal{S}$;

(ii) $\max \left\{D_{k}: \mu_{k} \neq 0\right\}=\max \left\{E_{j}: j \in \mathcal{S}\right\}$. 
We shall need still another reformulation of Pardue's formula for the regularity of a principal $p$-Borel ideal. Set $s=\max \left\{s_{k}: \mu_{k} \neq 0\right\}$, and for each $t$ with $1 \leq t \leq s$ let $m_{t}=\max \left\{k: \mu_{k t} \neq 0\right\}$. Finally set

$$
F_{t}=\sum_{i=t}^{s}\left(\sum_{k=2}^{n} \mu_{k i}\right) p^{i}+\left(m_{t}-1\right)\left(p^{t}-1\right) \quad \text { for all } t=1, \ldots, s .
$$

Proposition 1.6. With the notation introduced we have

$$
\max _{1 \leq t \leq s} F_{t}=\max _{j \in \mathcal{S}} E_{j}
$$

Proof. It is clear that $m_{j} \geq q_{j}$ for all $j \in \mathcal{S}$, so that $\max _{1 \leq t \leq s} F_{t} \geq \max _{j \in \mathcal{S}} E_{j}$.

In order to show the opposite inequality we first prove the following claim: Let $\mathcal{S}_{t}=\{j \in \mathcal{S}: j \geq t\}$ and $Q_{t}=\left\{q_{j}: j \in \mathcal{S}_{t}\right\}$. (Note that $\mathcal{S}_{t} \neq \emptyset$, since $s \in \mathcal{S}_{t}$.) Let $e \in \mathcal{S}_{t}$ such that $q_{e}=\max \left\{q_{j} \in Q_{t}\right\}$. Then we claim that $F_{e} \geq F_{t}$.

Indeed, we have

$$
F_{e}-F_{t}=-\sum_{i=t}^{e-1}\left(\sum_{k=2}^{n} \mu_{k i}\right) p^{i}+\left(m_{e}-1\right)\left(p^{e}-1\right)-\left(m_{t}-1\right)\left(p^{t}-1\right) .
$$

Since we assume that $q_{e}$ is maximal in $Q_{t}$ it follows that $\mu_{k i}=0$ for $k>q_{e}$ and $i \geq t$. Thus $m_{e}=q_{e} \geq \max \left\{k: \mu_{k t} \neq 0\right\}=m_{t}$, because again $\mu_{k t}=0$ for $k>q_{e}$.

Now it follows that

$$
F_{e}-F_{t}=-\sum_{i=t}^{e-1}\left(\sum_{k=2}^{q_{e}} \mu_{k i}\right) p^{i}+\left(q_{e}-1\right)\left(p^{e}-1\right)-\left(m_{t}-1\right)\left(p^{t}-1\right) .
$$

Finally, since $\sum_{i=t}^{e-1}\left(\sum_{k=2}^{q_{e}} \mu_{k i}\right) p^{i}=\sum_{k=2}^{q_{e}}\left(\sum_{i=t}^{e-1} \mu_{k i} p^{i}\right) \leq\left(q_{e}-1\right)\left(p^{e}-p^{t}\right)$, we get $F_{e}-F_{t} \geq\left(q_{e}-1\right)\left(p^{t}-1\right)-\left(m_{t}-1\right)\left(p^{t}-1\right)=\left(q_{e}-m_{t}\right)\left(p^{t}-1\right) \geq 0$. This concludes the proof of the claim.

Continuing with the proof of the opposite inequality, we let $t \leq s$ be the maximal number for which $F_{t}=\max _{1 \leq r \leq s} F_{r}$. Let $e \in \mathcal{S}_{t}$ be chosen such that $q_{e}$ is maximal in $Q_{t}$. Then, according to our claim, we have $F_{e} \geq F_{t}$. By the choice of $t$ this implies that $e=t$, so that, in particular, $t \in \mathcal{S}_{t}$. Since $q_{t}$ is maximal in $Q_{t}$ it now follows that $\mu_{k i}=0$ for $i \geq t$ and $k>q_{t}$. Consequently, $m_{t}=q_{t}$, and so $F_{t}=E_{t}$.

Remark 1.7. Using the methods of [1] the following result was proved in [5]: Let $\left(I_{t}\right)_{1 \leq t \leq s}$ be some stable ideals and $I=\prod_{t=1}^{s} I_{t}^{\left[p^{\left.r_{t}\right]}\right.}$ for some integers $0 \leq r_{1}<\cdots<$ $r_{s}$. If $I_{j}$ contains $x_{m\left(I_{j+1}\right)}^{p^{r_{j+1}-r_{j}}-1}$ for all $1 \leq j<s$ (we set $m(u)=\max \left\{j: x_{j} \mid u\right\}$ for a monomial $u$ ) and $\left.m\left(I_{j+1}\right)=\max \left\{m(u): u \in G\left(I_{j+1}\right)\right\}\right)$, then $\operatorname{reg}(I)=\operatorname{pa}(I)$, where

$$
\operatorname{pa}(I)=\max _{1 \leq t \leq s}\left\{\sum_{i>t}^{s} p^{r_{i}} \max \left(I_{i}\right)+\max _{u \in G\left(I_{t}\right)}\left[p^{r_{t}} \operatorname{deg}(u)+(m(u)-1)\left(p^{r_{t}}-1\right)\right]\right\} .
$$

Moreover if $I_{t}$ has the form $I_{t}=\prod_{i=2}^{n}\left(x_{1}, \ldots, x_{i}\right)^{\mu_{i t}}$ with $0 \leq \mu_{i t}<p$ for all $t<s$, the above result gives $\operatorname{reg}(I)=\max _{1 \leq t \leq s} F_{t}$. Hence the Pardue Conjecture holds in a special case, which can also be obtained directly from [1]. Trying to extend the equality $\operatorname{reg}(I)=\mathrm{pa}(I)$ for general products of $p^{i}$ th Frobenius powers of stable ideals one must consider first the following example which shows how tight Pardue's Conjecture is: Let $n=3, p=2, I_{1}=\left(x_{1}, x_{2}\right)^{2}, I_{2}=\left(x_{1}, x_{2}, x_{3}\right)$ and $I=I_{1} I_{2}^{[2]}$. Then $\operatorname{pa}(I)=4$, but reg $(I)>4$, because $I$ is not stable (see Proposition 2.1 below). 


\section{The Proof of PARdue's CONJecture}

In [1] it is shown that if $x^{\mu} \in S$ is a monomial which is not divisible by $x_{1}$, then

$$
\operatorname{reg}\left\langle x^{\mu}\right\rangle \geq \max _{k: \mu_{k} \neq 0}\left\{D_{k}\right\}
$$

In this section we will prove the opposite inequality. Our proof is based on the following result [4]:

Proposition 2.1 (Eisenbud, Reeves, Totaro). Let $I$ be a p-Borel ideal with $\max (I)=d$, and let $e \geq d$ be the smallest integer such that $I_{\geq e}$ is stable. Then $\operatorname{reg}(I)=e$.

Proposition 2.1 needs some explanation: $\max (I)=\max \{\operatorname{deg} u: u \in G(I)\}$, and $I_{\geq e}$ is the ideal generated by all monomials $u \in I$ with $\operatorname{deg} u \geq e$. Finally, recall that, according to Eliahou and Kervaire [3, a monomial ideal $I$ is stable if for all monomials (or equivalently all generators) $u$ of $I$ one has $\left(x_{i} / x_{m(u)}\right) u \in I$ for all $i \leq m(u)$, where $m(u)=\max \left\{j: x_{j} \mid u\right\}$.

Recall from Section 1 that $\left\langle x^{\mu}\right\rangle=\prod_{t} I_{t}^{\left[p^{t}\right]}$ where $I_{t}=\prod_{i=2}^{n}\left(x_{1}, \ldots, x_{i}\right)^{\mu_{i t}}$ with $0 \leq \mu_{i t}<p$. Thus the desired inequality will follow from Proposition 2.1 and

Theorem 2.2. For given integers $0 \leq r_{1}<\cdots<r_{s}$, and integers $0 \leq a_{t k}<$ $p^{r_{t+1}-r_{t}}$ for $t=1, \ldots, s$ and $k=1, \ldots, m_{t}$ let $I_{t}=\prod_{k=2}^{m_{t}}\left(x_{1}, \ldots, x_{k}\right)^{a_{t k}}$ and

$I=\prod_{t=1}^{s} I_{t}^{\left[p^{r_{t}}\right]}$. Let $\delta_{t}=\sum_{i=t}^{s} p^{r_{i}} \max \left(I_{i}\right)+\left(m_{t}-1\right)\left(p^{r_{t}}-1\right)$ and $d=\max \left\{\delta_{t} \mid 1 \leq\right.$ $t \leq s\}$. Then $I_{\geq d}$ is stable.

The proof needs some preparation.

Lemma 2.3. Let $J=\prod_{k=2}^{m}\left(x_{1}, \ldots, x_{k}\right)^{a_{k}}$ with $0 \leq a_{k} \leq p^{r}-1$, and let $\eta \in J$ be a monomial such that $\operatorname{deg} \eta \geq 1+\max \left\{m(\eta)\left(p^{r}-1\right), \max (J)+p^{r}-1\right\}$. Then there exists an integer $t$ such that $\eta \in x_{t}^{p^{r}} J$.

Proof. We reduce the problem to the case where $m(\eta) \leq m$. Since $J$ is stable, $\eta$ has the following Eliahou-Kervaire decomposition: $\eta=v w$ for monomials $v$ and $w$ with $v \in G(J)$ and $\min (w) \geq m(v)$. We may assume that $w \notin\left(x_{t}^{p^{r}}\right)$ for all $t$. Then $w=w^{\prime} x_{m+1}^{\beta_{m+1}} \cdots x_{m(\eta)}^{\beta_{m(\eta)}}$ with $\beta_{i} \leq p^{r}-1$. Thus the element $\eta^{\prime}=v w^{\prime}$ has degree $\geq \operatorname{deg} \eta-(m(\eta)-m)\left(p^{r}-1\right) \geq m\left(p^{r}-1\right)+1$. Since $m\left(\eta^{\prime}\right) \leq m$ and $\max (J) \leq(m-1)\left(p^{r}-1\right)$, we may replace $\eta$ by $\eta^{\prime}$, and thus may as well suppose that $m(\eta) \leq m$.

Let $\eta=x_{1}^{\alpha_{1}} \cdots x_{m(\eta)}^{\alpha_{m(\eta)}}$. We apply induction on $\max (J)$, and may assume that $a_{m} \neq 0$. If $\max (J)=1$, then $J=\left(x_{1}, \ldots, x_{m}\right)$. If $m(\eta)=1$, then $\eta=x_{1}^{\alpha_{1}}$ with $\alpha_{1} \geq \max (J)+p^{r}=p^{r}+1$. In that case, $\eta=x_{1}^{p^{r}} \eta^{\prime}$ with $\eta^{\prime}=x_{1}^{\alpha_{1}-p^{r}} \in J$. Suppose now that $m(\eta) \geq 2$. Then, since $\operatorname{deg} \eta \geq m(\eta)\left(p^{r}-1\right)+1$, it follows that $\eta \in\left(x_{t}^{p^{r}}\right)$ for a certain $t \leq m(\eta)$, and so $\eta=x_{t}^{p^{r}} \eta^{\prime}$ where $\eta^{\prime}$ is a monomial of degree $\geq(m(\eta)-1)\left(p^{r}-1\right)>1$. Hence $\eta^{\prime} \in J$, and so $\eta \in x_{t}^{p^{r}} J$.

Now suppose that $\max (J)>1$. We will distinguish several cases. In the first case suppose that $\alpha_{m(\eta)} \geq p^{r}$. Let again $\eta=v w$ be the Eliahou-Kervaire decomposition of $\eta$. Then $\operatorname{deg} w=\operatorname{deg} \eta-\operatorname{deg} v \geq p^{r}>0$. Hence, since $m(v) \leq \min (w)$, it follows that $x_{m(\eta)}^{p^{r}}$ divides $w$, and we are done.

Now we consider the case that $\alpha_{m(\eta)} \leq p^{r}-1, m(\eta) \geq 3$ and $\alpha_{m(\eta)} \leq \sum_{i=m(\eta)}^{m} a_{i}$. We choose the maximal integer $t, m(\eta) \leq t \leq m$, such that $\alpha_{m(\eta)} \leq \sum_{i=t}^{m} a_{i}$, and 
write $\alpha_{m(\eta)}=\sum_{i=t+1}^{m} a_{i}+b_{t}$ with $1 \leq b_{t} \leq a_{t}$. Now set $\varphi=\eta / x_{m(\eta)}^{\alpha_{m(\eta)}}$. Observe that for all monomials $\rho \in J$ with $m(\rho) \leq m$ one has

$$
\rho / x_{m(\eta)} \in \prod_{k=2}^{m-1}\left(x_{1}, \ldots, x_{k}\right)^{a_{k}}\left(x_{1}, \ldots, x_{m}\right)^{a_{m}-1} .
$$

Applying (1) successively we see that $\varphi \in J^{\prime \prime}$ where

$$
J^{\prime \prime}=\prod_{k=2}^{t-1}\left(x_{1}, \ldots, x_{k}\right)^{a_{k}}\left(x_{1}, \ldots, x_{t}\right)^{a_{t}-b_{t}} .
$$

We have

$\operatorname{deg} \varphi=\operatorname{deg} \eta-\alpha_{m(\eta)} \geq \operatorname{deg} \eta-\left(p^{r}-1\right) \geq 1+(m(\eta)-1)\left(p^{r}-1\right) \geq 1+m(\varphi)\left(p^{r}-1\right)$, and $\operatorname{deg} \varphi=\operatorname{deg} \eta-\alpha_{m(\eta)} \geq \max (J)+p^{r}-\alpha_{m(\varphi)}=\max \left(J^{\prime \prime}\right)+p^{r}$. Hence we may apply our induction hypothesis and conclude that there exists an integer $q \leq m(\varphi)$ such that $\varphi \in x_{q}^{p^{r}} J^{\prime \prime}$. It follows that $\eta \in x_{q}^{p^{r}} J$.

Next we consider the case $\alpha_{m(\eta)} \leq p^{r}-1, m(\eta) \geq 3$, and $\alpha_{m(\eta)}>\sum_{i=m(\eta)}^{m} a_{i}$. Using (1) again we see that $\eta=x_{m(\eta)}^{\sum_{i=m(\eta)}^{m}{ }^{a_{i}}} \eta^{\prime}$ with $\eta^{\prime} \in \tilde{J}$ where

$$
\tilde{J}=\prod_{k=2}^{m(\eta)-1}\left(x_{1}, \ldots, x_{k}\right)^{a_{k}} .
$$

Note that for any monomial $\rho \in J$ with $m(\rho)>m$ it follows that $\rho / x_{m(\eta)} \in J$. Applying this successively to $\eta^{\prime}$ we see that $\varphi=\eta / x_{m(\eta)}^{\alpha_{m(\eta)}}$ belongs to $\tilde{J}$. As in the second case it follows that $\operatorname{deg} \varphi \geq 1+m(\varphi)\left(p^{r}-1\right)$. Since on the other hand $\max (\tilde{J}) \leq(m(\eta)-2)\left(p^{r}-1\right)$, it also follows that $\operatorname{deg} \varphi \geq 1+(m(\eta)-1)\left(p^{r}-1\right) \geq$ $\max \tilde{J}+p^{r}$. Applying the induction hypothesis to $\varphi$ and $\tilde{J}$ yields the desired conclusion for $\eta$.

It remains to consider the case $\alpha_{m(\eta)} \leq p^{r}-1$ and $m(\eta) \leq 2$. If $m(\eta)=1$, then $\alpha_{1} \geq p^{r}$, a contradiction. Therefore $\eta=x_{1}^{\alpha_{1}} x_{2}^{\alpha_{2}}$ with $\alpha_{2} \neq 0$ and $\alpha_{1}+\alpha_{2} \geq$ $\max \left\{2 p^{r}-1, \max J+p^{r}\right\}$. It follows that $\alpha_{1} \geq p^{r}$. Then the element $\eta^{\prime}=x_{1}^{\alpha_{1}-p^{r}} x_{2}^{\alpha_{2}}$ belongs to $\left(x_{1}, x_{2}\right)^{\max J}$ which is contained in $J$, and so $\eta=x_{1}^{p^{r}} \eta^{\prime} \in x_{1}^{p^{r}} J$.

Corollary 2.4. Let $J=\prod_{k=2}^{m}\left(x_{1}, \ldots, x_{k}\right)^{a_{k}}$ where $0 \leq a_{k} \leq p^{r}-1$ for $k=$ $2, \ldots, m$, and let $q$ be a positive integer and $\eta \in J$ a monomial with $m(\eta)<q$ and $\operatorname{deg} \eta \geq 1+\max \left\{(q-1)\left(p^{r}-1\right), \max (J)\right\}$. Then there exists $t \leq m(\eta)$ such that $x_{q}^{p^{r}-1} \eta \in x_{t}^{p^{r}} J$.

Proof. Let $\eta^{\prime}=x_{q}^{p^{r}-1} \eta$. We have $\operatorname{deg} \eta^{\prime} \geq 1+\max \left\{m\left(\eta^{\prime}\right)\left(p^{r}-1\right), \max (J)+p^{r}-1\right\}$ since $m\left(\eta^{\prime}\right)=q$. Thus by Lemma 2.3 there exists an integer $t \leq m\left(\eta^{\prime}\right)=q$ such that $\eta^{\prime} \in x_{t}^{p^{r}} J$, and hence $x_{q}^{p^{r}-1} \eta \in x_{t}^{p^{r}} J$. Since $p^{r}-1$ is the maximal power of $x_{q}$ which divides $\eta^{\prime}$, we have $t \neq q$ and so $t \leq m(\eta)$.

Lemma 2.5. Let $J=\prod_{k=2}^{m}\left(x_{1}, \ldots, x_{k}\right)^{a_{k}}$ with $0 \leq a_{k} \leq p^{r-e}-1$ for $k=2, \ldots, m$ and integers $0 \leq e<r$. Let $I=J^{\left[p^{e}\right]}$ and $\eta \in I$ be a monomial such that $\operatorname{deg} \eta \geq$ $1+\max \left\{m(\eta)\left(p^{r}-1\right), \max (I)+p^{r}-p^{e}+m(\eta)\left(p^{e}-1\right)\right\}$. Then there exists $t \leq m(\eta)$, such that $\eta \in x_{t}^{p^{r}} I$. 
Proof. We may write $\eta=v^{p^{e}} w, v \in G(J)$ and $w=\sigma_{1}^{p^{e}} \sigma_{0}$, where $\sigma_{0}$ and $\sigma_{1}$ are monomials, and $\sigma_{0} \notin\left(x_{1}^{p^{e}}, \ldots, x_{m(\eta)}^{p^{e}}\right)$. Thus $\operatorname{deg} \sigma_{0} \leq m(\eta)\left(p^{e}-1\right)$ and the monomial $\eta^{\prime}=v \sigma_{1}$ belongs to $J$. Since $\eta=\eta^{\prime p^{e}} \sigma_{0}$ it follows that

$$
\begin{aligned}
p^{e} \operatorname{deg} \eta^{\prime} & =\operatorname{deg} \eta-\operatorname{deg} \sigma_{0} \geq \operatorname{deg} \eta-m(\eta)\left(p^{e}-1\right) \\
& \geq 1+\max \left\{m(\eta)\left(p^{r}-p^{e}\right), p^{e} \max (J)+p^{r}-p^{e}\right\} .
\end{aligned}
$$

Therefore $\operatorname{deg} \eta^{\prime} \geq\left(1 / p^{e}\right)+\max \left\{m(\eta)\left(p^{r-e}-1\right), \max (J)+p^{r-e}-1\right\}$. Since $\operatorname{deg} \eta^{\prime}$ is an integer we get $\operatorname{deg} \eta^{\prime} \geq 1+\max \left\{m(\eta)\left(p^{r-e}-1\right), \max (J)+p^{r-e}-1\right\}$. Note that $m\left(\eta^{\prime}\right) \leq m(\eta)$. Therefore by Lemma 2.3 there exists an integer $t, t \leq m\left(\eta^{\prime}\right) \leq m(\eta)$, such that $\eta^{\prime} \in x_{t}^{p^{r-e}} J$. Thus $\eta \in x_{t}^{p^{r}} I$.

Applying Lemma 2.5 recursively we get

Corollary 2.6. With the hypotheses of Lemma 2.5 suppose in addition that $\operatorname{deg} \eta \geq$ $c p^{r}+1+\max \left\{m(\eta)\left(p^{r}-1\right), \max (I)+p^{r}-p^{e}+m(\eta)\left(p^{e}-1\right)\right\}$ for some integer $c \geq 0$. Then there exists a monomial $\sigma$ of degree $c+1$ such that $m(\sigma) \leq m(\eta)$ and $\eta \in \sigma^{p^{r}} I$.

Lemma 2.7. Let $J=\prod_{k=2}^{m}\left(x_{1}, \ldots, x_{k}\right)^{a_{k}}$ with $0 \leq a_{k} \leq p^{r-e}-1$ for $k=2, \ldots, m$ and integers $0 \leq e<r$. Let $I=J^{\left[p^{e}\right]}$, and let $q$ be a positive integer and $\eta \in J$ a monomial with $m(\eta)<q$ and $\operatorname{deg} \eta \geq c p^{r}+1+\max \left\{(q-1)\left(p^{r}-1\right), \max (I)+\right.$ $\left.(q-1)\left(p^{e}-1\right)\right\}$ for some integer $c \geq 0$. Then there exists a monomial of degree $c+1$ such that $x_{q}^{p^{r}-1} \eta \in \sigma^{p^{r}} I$ and $m(\sigma) \leq m(\eta)$.

Proof. Set $\eta^{\prime}=x_{q}^{p^{r}-1} \eta$. Then

$\operatorname{deg} \eta^{\prime} \geq c p^{r}+1+\max \left\{m\left(\eta^{\prime}\right)\left(p^{r}-1\right), \max (I)+p^{r}-p^{e}+m\left(\eta^{\prime}\right)\left(p^{e}-1\right)\right\}$.

So by Corollary 2.6 there exists a monomial $\sigma$ with $m(\sigma) \leq m\left(\eta^{\prime}\right)$ and $\operatorname{deg} \sigma=c+1$ such that $\eta^{\prime} \in \sigma^{p^{r}} I$. Finally we have $m(\sigma) \leq m(\eta)$ because $x_{q}$ does not divide $\sigma$ since it appears only with power $p^{r}-1$ in $\sigma$.

Lemma 2.8. Let $I_{t}=\prod_{k=2}^{m_{t}}\left(x_{1}, \ldots, x_{k}\right)^{a_{t k}}$ with $0 \leq a_{t k} \leq p^{e_{t+1}-e_{t}}-1,1 \leq$ $t \leq s$, and integers $0 \leq e_{1}<\cdots<e_{s}<r=e_{s+1}$. Let $I=\prod_{t=1}^{s} I_{t}^{\left[p^{e^{t}}\right]}$, and let $q$ be a positive integer and $\eta \in I$ a monomial with $m(\eta)<q$ and $\operatorname{deg} \eta \geq$ $c p^{r}+1+\max _{1 \leq t \leq s+1}\left\{\sum_{i=t}^{s} p^{e_{i}} \max \left(I_{i}\right)+(q-1)\left(p^{e_{t}}-1\right)\right\}$ for some integer $c \geq 0$. (Here $\sum_{i=t}^{s} p^{e_{i}} \max \left(I_{i}\right)=0$ for $t=s+1$.) Then there exists a monomial $\sigma$ with $\operatorname{deg} \sigma=c+1, m(\sigma) \leq m(\eta)$ and $x_{q}^{p^{r}-1} \eta \in \sigma^{p^{r}} I$.

Proof. We apply induction on $s$. The case $s=1$ is given in Lemma 2.7 Let $d_{j}=\sum_{i=j}^{s} p^{e_{i}} \max \left(I_{i}\right)+(q-1)\left(p^{e_{j}}-1\right), 1 \leq j \leq s+1$, and let $t \leq s$ be a maximal integer such that $d_{t}=\max \left\{d_{j}: 1 \leq j \leq s\right\}$. Then we have $d_{j}<d_{t}$ for $t<j \leq s$ and $d_{j} \leq d_{t}$ for $j<t$.

We now distinguish two cases. In case $t>1$, write $\eta=\eta^{\prime} \prod_{i=t}^{s} v_{i}^{p^{e_{i}}}$ with $v_{i} \in$ $G\left(I_{i}\right)$ and $\eta^{\prime} \in I^{\prime}=\prod_{i=1}^{t-1} I_{i}^{\left[p^{e_{i}}\right]}$. We have $\operatorname{deg}\left(\eta^{\prime}\right)=\operatorname{deg}(\eta)-\sum_{i=t}^{s} p^{e_{i}} \max \left(I_{i}\right) \geq$ $c p^{r}+1+d_{t}-\sum_{i=t}^{s} p^{e_{i}} \max \left(I_{i}\right)=c p^{r}+1+(q-1)\left(p^{e_{t}}-1\right)$. Choose the maximal integer $\varepsilon \geq 0$ such that $\operatorname{deg}\left(\eta^{\prime}\right) \geq c p^{r}+1+\varepsilon p^{e_{t}}+(q-1)\left(p^{e_{t}}-1\right)$. As $d_{t}=\max \left\{d_{i}: 1 \leq i \leq s\right\}$, we see that $\eta^{\prime}$ satisfies the necessary inequalities and so by induction hypothesis there exists a monomial $\tau$ with $\operatorname{deg}(\tau)=c p^{r-e_{t}}+\varepsilon+1, m(\tau) \leq m\left(\eta^{\prime}\right) \leq m(\eta)$ and $x_{q}^{p^{e} t}-1 \eta^{\prime}=\tau^{p^{e t}} \eta^{\prime \prime}$ for some $\eta^{\prime \prime} \in I^{\prime}$. Note that by the choice of $\varepsilon$, we have $\operatorname{deg}\left(\eta^{\prime}\right) \leq$ $c p^{r}+1+\varepsilon p^{e_{t}}+q\left(p^{e_{t}}-1\right)$, and so $p^{e_{t}} \operatorname{deg}(\tau)+\operatorname{deg}\left(\eta^{\prime \prime}\right) \leq c p^{r}+1+\varepsilon p^{e_{t}}+(q+1)\left(p^{e_{t}}-1\right)$. Hence $\operatorname{deg}\left(\eta^{\prime \prime}\right) \leq 1-p^{e_{t}}+(q+1)\left(p^{e_{t}}-1\right)=q\left(p^{e_{t}}-1\right)$. 
Set $\rho=\tau \prod_{i=t}^{s} v_{i}^{p^{e_{i}-e_{t}}} ;$ then $\rho \in \tilde{I}=\prod_{i=t}^{s} I_{i}^{\left[p^{e_{i}-e_{t}}\right]}$, and $p^{e_{t}} \operatorname{deg}(\rho)=\operatorname{deg}\left(x_{q}^{p^{e_{t}}-1} \eta\right)$ $-\operatorname{deg}\left(\eta^{\prime \prime}\right) \geq c p^{r}+p^{e_{t}}+\max \left\{d_{t}, d_{s+1}\right\}-q\left(p^{e_{t}}-1\right)=c p^{r}+1+a$ for $a=p^{e_{t}} b$ and $b=\max \left\{\sum_{j=i}^{s} p^{e_{j}-e_{t}} \max \left(I_{j}\right)+(q-1)\left(p^{e_{i}-e_{t}}-1\right): t \leq i \leq s+1\right\}$. Therefore $\operatorname{deg}(\rho) \geq c p^{r-e_{t}}+1 / p^{e_{t}}+b$. Using that $\operatorname{deg} \rho$ is an integer we get $\operatorname{deg}(\rho) \geq$ $c p^{r-e_{t}}+1+b$. Thus we may apply the induction hypothesis to $\rho, \tilde{I}$, and hence there exists a monomial $\sigma$ with $m(\sigma) \leq m(\rho) \leq m(\eta), \operatorname{deg}(\sigma)=c+1$ such that $x_{q}^{p^{r-e_{t}}-1} \rho \in \sigma^{p^{r-e_{t}}} \tilde{I}$. Hence $x_{q}^{p^{r}-1} \eta=x_{q}^{p^{r}-p^{e_{t}}} \rho^{p^{e_{t}}} \eta^{\prime \prime} \in \sigma^{p^{r}} I$.

Finally we consider the case $t=1$, and write $\eta=\varphi \prod_{i=2}^{s} v_{i}^{p^{e_{i}}}, v_{i} \in G\left(I_{i}\right)$ and $\varphi \in I_{1}^{\left[p^{e_{1}}\right]}$. We have $\operatorname{deg}(\varphi)=\operatorname{deg}(\eta)-\sum_{i=2}^{s} p^{e_{i}} \max \left(I_{i}\right) \geq c p^{r}+1+d_{1}-$ $\sum_{i=2}^{s} p^{e_{i}} \max \left(I_{i}\right)=c p^{r}+1+p^{e_{1}} \max \left(I_{1}\right)+(q-1)\left(p^{e_{1}}-1\right)$. However, $t=1$ implies $d_{2}<d_{1}$, and so $p^{e_{1}} \max \left(I_{1}\right)+(q-1)\left(p^{e_{1}}-1\right)>(q-1)\left(p^{e_{2}}-1\right)$. Thus $\varphi$ satisfies the condition of Lemma 2.7. As in the previous case we choose the maximal integer $\varepsilon \geq 0$ such that $\operatorname{deg}(\varphi) \geq c p^{r}+1+\varepsilon p^{e_{2}}+(q-1)\left(p^{e_{2}}-1\right)$. Then by Lemma 2.7 there exists a monomial $\gamma$ with $\operatorname{deg}(\gamma)=c p^{r-e_{2}}+1+\varepsilon, m(\gamma) \leq m(\eta)$ and $x_{q}^{p^{e_{2}}-1} \varphi=\gamma^{p^{e_{2}}} \varphi^{\prime}$ for some $\varphi^{\prime} \in I_{1}^{\left[p^{e_{1}}\right]}$, and we see as above that $\operatorname{deg}\left(\varphi^{\prime}\right) \leq q\left(p^{e_{2}}-1\right)$.

Set $\psi=\gamma^{\prime} \prod_{i=2}^{s} v_{i}^{p^{e_{i}-e_{2}}} ;$ then $\psi \in \hat{I}=\prod_{i=2}^{s} I_{i}^{\left[p^{e_{i}-e_{2}}\right]}, m(\psi) \leq m(\eta)$, and

$$
\begin{aligned}
p^{e_{2}} \operatorname{deg}(\psi) & =\operatorname{deg}\left(x_{q}^{p^{e_{2}}-1} \eta\right)-\operatorname{deg}\left(\varphi^{\prime}\right) \\
& \geq c p^{r}+p^{e_{2}}+\max \left\{d_{j}: 2 \leq j \leq s+1\right\}-q\left(p^{e_{2}}-1\right) \\
& =c p^{r}+1+\max \left\{\sum_{i=j}^{s} p^{e_{i}} \max \left(I_{i}\right)+(q-1)\left(p^{e_{j}}-p^{e_{2}}\right): 2 \leq j \leq s+1\right\} .
\end{aligned}
$$

Since $\operatorname{deg} \psi$ is an integer we get

$$
\begin{aligned}
\operatorname{deg}(\psi) \geq & c p^{r-e_{2}}+1 \\
& +\max \left\{\sum_{i=j}^{s} p^{e_{i}-e_{2}} \max \left(I_{i}\right)+(q-1)\left(p^{e_{j}-e_{2}}-1\right): 2 \leq j \leq s+1\right\} .
\end{aligned}
$$

Thus we may apply our induction hypothesis to $\psi$ and $\hat{I}$ and conclude that $x_{q}^{p^{r-e_{2}}-1} \psi \in \nu^{p^{r-e_{2}}} \hat{I}$ for some monomial $\nu$ with $\operatorname{deg}(\nu)=c+1$ and $m(\nu) \leq m(\psi) \leq$ $m(\eta)$. This yields the desired conclusion.

We are now in the position to prove Theorem 2.2 .

Proof of 2.2. Let $\rho=\prod_{t=1}^{s} u_{t}^{p^{r_{t}}} w, u_{t} \in G\left(I_{t}\right)$ and $w$ is a monomial such that $\operatorname{deg}(\rho)=d$. Let $j<m(\rho)$. We must show that $x_{j} \rho / x_{m(\rho)} \in I_{\geq d}$. Apply induction on $s$, case $s=0$ being trivial. If $m(\rho)=m(\eta), \eta=\prod_{t=1}^{s-1} u_{t}^{p^{r t}} w$, then we may apply the induction hypothesis because $\operatorname{deg}(\eta)=d-\operatorname{deg}\left(u_{s}^{p^{\bar{r}_{s}}}\right) \geq \max \left\{\delta_{t}^{\prime}: 1 \leq t \leq s-1\right\}$ for $\delta_{t}^{\prime}=\sum_{i=t}^{s-1} p^{r_{i}} \max \left(I_{i}\right)+\left(m_{t}-1\right)\left(p^{r_{t}}-1\right)$. By the induction hypothesis $I^{\prime}=$ $\prod_{t=1}^{s-1} I_{t}^{\left[p^{r_{t}}\right]} \operatorname{has} I_{\geq \operatorname{deg}(\eta)}^{\prime}$ stable and so $\left.x_{j} \eta / x_{m(\eta)}\right) \in I_{\geq \operatorname{deg}(\eta)}^{\prime}$. Hence $x_{j} \rho / x_{m(\rho)}=$ $\left(x_{j} \eta / x_{m(\eta)}\right) u_{s}^{p^{r_{s}}} \in I_{\geq d}$.

We may suppose from now on that $m(\rho)=m\left(u_{s}\right)>m(\eta)$. Set $q=m\left(u_{s}\right)$, $d_{t}=\sum_{i=t}^{s-1} p^{r_{i}} \max \left(I_{i}\right)+(q-1)\left(p^{r_{t}}-1\right)$. We have $\delta_{t}^{\prime} \geq d_{t}$ if and only if $m_{t} \geq q$. In particular $\delta_{s}^{\prime} \geq d_{s}$ since $m_{s} \geq q$. If $m_{t}<q$, then $\max \left(I_{t}\right) \leq(q-1)\left(p^{r_{t+1}-r_{t}}-1\right)$ since $a_{t k} \leq p^{r_{t+1}-r_{t}}-1$, and so $d_{t+1}-\delta_{t}^{\prime}=(q-1)\left(p^{r_{t+1}}-1\right)-\left(m_{t}-1\right)\left(p^{r_{t}}-1\right)-$ $p^{r_{t}} \max \left(I_{t}\right)>(q-1)\left(p^{r_{t+1}}-p^{r_{t}}\right)-p^{r_{t}} \max \left(I_{t}\right) \geq 0$. Thus $\delta_{t}^{\prime}<d_{t+1}$ if $m_{t}<q$. The same argument shows that $d_{t} \leq d_{t+1}$ if $m_{t}<q$. 
By backwards induction on $j$ we now show that

$$
\max \left\{d_{t}: j \leq t \leq s\right\} \leq \max \left\{\delta_{t}^{\prime}: j \leq t \leq s\right\} .
$$

We have already seen that (2) holds for $j=s$. Suppose the inequality holds for $j+1$. If $m_{j} \geq q$, then $\delta_{j}^{\prime} \geq d_{j}$, and so (2) is implied by the induction hypothesis. If $m_{j}<q$, then $\delta_{j}^{\prime}<d_{j+1}, d_{j} \leq d_{j+1}$, and hence $\delta_{j}^{\prime}<d_{j+1} \leq \max \left\{d_{t}: j+1 \leq t \leq\right.$ $s\} \leq \max \left\{\delta_{t}^{\prime}: j+1 \leq t \leq s\right\}$. Hence $\max \left\{\delta_{t}^{\prime}: j \leq t \leq s\right\}=\max \left\{\delta_{t}^{\prime}: j+1 \leq t \leq\right.$ $s\} \geq \max \left\{d_{t}: j+1 \leq t \leq s\right\}=\max \left\{d_{t}: j \leq t \leq s\right\}$, as desired.

Now since by (2) we have $\operatorname{deg}\left(x_{j} \eta\right)=1+\operatorname{deg}(\eta)=1+\operatorname{deg}(\rho)-p^{r_{s}} \max \left(I_{s}\right)=$ $1+\max \left\{\delta_{t}^{\prime}: 1 \leq t \leq s\right\} \geq 1+\max \left\{d_{t}: 1 \leq t \leq s\right\}$, we may apply Lemma 2.8 for $s-1, x_{j} \eta \in I^{\prime}$ and $q$. Then there exists $e \leq m(\eta)$ such that $x_{q}^{p^{r_{s}}-1}\left(x_{j} \eta\right)=x_{e}^{p^{r_{s}}} \eta^{\prime}$ for some $\eta^{\prime} \in I^{\prime}$. Thus $x_{j} \rho / x_{m(\rho)}=\left(x_{e} u_{s} / x_{m\left(u_{s}\right)}\right)^{p^{r_{s}}} \eta^{\prime}$ belongs to $I$, because $I_{s}$ is stable.

For a monomial $u$, we set $\operatorname{pa}(u)=\max _{k}: \mu_{k} \neq 0\left\{D_{k}\right\}$ if $u$ is not a multiple of $x_{1}$. Otherwise $u=x_{1}^{\mu_{1}} v$ such that $v \notin\left(x_{1}\right)$, and we set pa $(u)=\mu_{1}+\mathrm{pa}(v)$ (cf. Section 1). By our main theorem we have $\operatorname{reg}\langle u\rangle=\mathrm{pa}(u)$. More generally we get

Corollary 2.9. Let I be a p-Borel ideal with Borel generators $u_{1}, \ldots, u_{m}$. Then

$$
\operatorname{reg}(I) \leq \max \left\{\mathrm{pa}\left(u_{1}\right), \ldots, \operatorname{pa}\left(u_{m}\right)\right\},
$$

and equality holds if $I$ is principal p-Borel.

Proof. For each $i=1, \ldots, m$, the ideal $\left\langle u_{i}\right\rangle_{\geq d}$ is stable for $d \geq \operatorname{pa}\left(u_{i}\right)$. Thus $I_{\geq d}$ is stable for $d \geq \max \left\{\mathrm{pa}\left(u_{1}\right), \ldots, \mathrm{pa}\left(u_{m}\right)\right\}$. Therefore the assertion follows from Proposition 2.1.

\section{REFERENCES}

[1] A. Aramova and J. Herzog, p-Borel principal ideals, Illinois J. Math. 41, no. 1 (1997), 103-121. MR 98i: 13020

[2] D. Bayer and M. Stillman, A criterion for detecting $m$-regularity, Invent. Math. 87 (1987), 1-11. MR 87k:13019

[3] S. Eliahou and M. Kervaire, Minimal resolutions of some monomial ideals, J. of Algebra 129 (1990) 1-25. MR 91b:13019

[4] D. Eisenbud, A. Reeves and B. Totaro, Initial ideals, Veronese subrings, and rates of algebras, Adv Math. 109 (1994), 168-187. MR 96d:13030

[5] V. Ene, G. Pfister and D. Popescu, Betti numbers for p-stable ideals, Comm. Algebra 28 (2000), 1515-1531. CMP 2000:08

[6] K. Pardue, Nonstandard Borel-fixed ideals, Dissertation, Brandeis University, 1994.

FB6 Mathematik und Informatik, Universität - GHS - Essen, Postfach 103764, 45117 Essen, Germany

E-mail address: juergen.herzog@uni-essen.de

Institute of Mathematics, University of Bucharest, P.O. Box 1-764, Bucharest 70700, ROMANIA

E-mail address: dorin@stoilow.imar.ro 